\title{
Increased expression of claudin-12 promotes the metastatic phenotype of human bronchial epithelial cells and is associated with poor prognosis in lung squamous cell carcinoma
}

\author{
LEMENG SUN $^{1}$, LIANGSHU FENG ${ }^{2}$ and JIUWEI CUI ${ }^{1}$ \\ ${ }^{1}$ Department of Oncology, Stem Cell and Cancer Center, The First Bethune Hospital, Jilin University; \\ ${ }^{2}$ Department of Neurology and Neuroscience Center, First Hospital of Jilin University, Changchun, Jilin 130021, P.R. China
}

Received May 6, 2018; Accepted October 19, 2018

DOI: $10.3892 /$ etm.2018.6964

\begin{abstract}
A prior study by our group using cDNA array analysis identified the tight junction component claudin-12 (CLDN12) to be an upregulated gene in lung squamous cell carcinoma (SqCC) cells compared with normal human bronchial epithelial cells. The present study aimed to explore the effect and underlying molecular mechanism of CLDN12 with regard to the malignant phenotype of SqCC. Firstly, the expression patterns of CLDN12 in SqCC tissues, lung adenocarcinoma tissues and histologically non-neoplastic lung epithelial tissues were investigated by immunohistochemistry and western blotting. Additionally, associations between CLDN12 expression and clinicopathological indicators were examined in patients with SqCC. Furthermore, the impact of CLDN12 on the malignant phenotype of the human bronchial epithelial cell line BEAS-2B in vitro was assessed using the Cell Counting kit-8 assay, Transwell assay and a wound-healing experiment. Western blotting and immunofluorescence were also used to detect the impact of CLDN12 on the epithelial-mesenchymal transition (EMT) of BEAS-2B cells. Tyrosine kinase 2 (Tyk2) RNA interference was further utilized to determine the impact of the Tyk2/signal transducer and activator of transcription 1 (Stat1) signaling pathway on the EMT of BEAS-2B cells. To conclude, it was indicated that the expression of CLDN12 was upregulated in $\mathrm{SqCC}$ tissues and was associated with the extent of lymphatic metastasis in patients with SqCC. Furthermore, CLDN12 promoted the EMT of human bronchial epithelial
\end{abstract}

Correspondence to: Dr Jiuwei Cui, Department of Oncology, Stem Cell and Cancer Center, The First Bethune Hospital, Jilin University, 71 Xinmin Street, Changchun, Jilin 130021, P.R. China E-mail: cjwjlu@126.com

Abbreviations: TJ, Tight junction; CLDNs, claudins; EMT, epithelial-mesenchymal transition; SqCC, lung squamous cell carcinoma; CCK8, Cell Counting kit-8; OC, ovarian cancer

Key words: tight junction, claudin-12, epithelial-mesenchymal transition, lung squamous cell carcinoma, metastasis cells in vitro. The findings indicated that the induction of Tyk2/Stat1 signaling appears to be an important mechanism by which CLDN12 promotes the EMT of SqCC cells.

\section{Introduction}

It is well established that $>90 \%$ of fatalities associated with solid tumors are primarily due to tumor metastasis (1). Lung cancer has been revealed to be the leading cause of cancer-associated fatality, with 5-year survival rates reportedly as low as $15 \%$ (2). Hence, the molecular mechanism of metastasis in the tumorigenesis of lung cancer is of key research significance. Recently, an increasing number of studies have indicated that the altered expression of claudin (CLDN) tight junction (TJ) proteins has an essential role in the tumorigenesis and progression of various human cancer types by affecting the structure and function of TJs and associated cellular signaling pathways $(3,4)$. TJs are located at the apex of cell junctions, and function to regulate cell adhesion and maintain cell polarity and permeability (5). CLDNs are the key proteins that comprise TJs, and evidence has suggested that the expression and localization of CLDNs is frequently altered in tumor cells (6). For instance, it was indicated that CLDN1 was overexpressed in colorectal cancer (CRC) compared with in normal mucosa, and CLDN1 targeting with an anti-CLDN1 monoclonal antibody resulted in decreased growth and survival of CRC cells, suggesting that CLDN1 could be a novel potential therapeutic target in CRC (7). In addition, high-level cytoplasmic CLDN3 expression was revealed to be an independent predictor of poor survival in triple-negative breast cancer (8). Furthermore, CLDN4 has been reported to be overexpressed in advanced ovarian cancer (OC), and Kaplan-Meier survival analysis and Log-Rank testing suggested that high expression of CLDN4 may have prognostic value in OC (9). These observations indicate that alterations in the expression of CLDNs may be associated with tumorigenesis and cancer progression in various human carcinomas.

The distinct expression patterns of CLDNs in various cancer types present an opportunity for application in the diagnosis and treatment of tumors, and in determining the mechanisms of different therapeutic responses. It has been 
revealed that the expression and function of CLDNs may be highly tissue-specific and depend on the active molecular pathways in epithelial cells (4). Previous genetic and pharmacological assays confirmed that the expression of CLDN3 was downregulated in colon cancer, and that loss of CLDN3 induced $\mathrm{Wnt} / \beta$-catenin activation and thus promoted colon cancer (10). However, the expression pattern of claudin-12 (CLDN12) and its association with prognostic value in human squamous cell carcinoma ( $\mathrm{SqCC}$ ) is not well established. Therefore, the aim of the present study was to determine the expression levels and prognostic value of CLDN12 in SqCC tissues, as well as the impact and mechanism of CLDN12 with regards to the tumorigenesis and progression of $\mathrm{SqCC}$, in order to identify novel targets to be utilized in the treatment of SqCC and control of early metastasis.

\section{Patients and methods}

Patients. The SqCC sections were collected via surgery from 50 patients (27 males, 23 females; age range, 43-74 years; median age, 59 years) who were treated at The First Bethune Hospital, Jilin University (Changchun, China) between June 2005 and July 2012, who had pathologically confirmed diagnoses of SqCC. The cases were selected based on the following criteria: Pathologically confirmed diagnosis of SqCC; no previous malignant disease or second primary tumor; and no history of radiotherapy and chemotherapy. All patients with SqCC were graded and classified according to the American Joint Committee on Cancer tumor node metastasis (TNM) staging system. Additionally, 10 cases of lung adenocarcinoma tissues (6 males, 4 females; age range, 49-69 years; median age, 67 years) were collected from patients who were treated at The First Bethune Hospital, Jilin University between October 2007 and May 2013. A further 20 sections of histologically non-neoplastic lung tissues were also obtained from patients with pneumonia (11 males, 9 females; age range, 45-67 years; median age, 52 years). The medical records of the patients were reviewed to determine clinical and pathological characteristics.

Immunohistochemistry. An immunohistochemistry assay was used to detect the expression of CLDN12 and epithelial (E)-Cadherin in the tissue samples from the 50 cases of SqCC, 10 cases of lung adenocarcinoma and 20 cases of non-neoplastic mucosa. Patients who had smoked $<100$ cigarettes in their lifetime were defined as non-smokers. No neoadjuvant therapy was applied in any of the 50 patients with SqCC. The pretreatment for the sections were performed as follows: fixed in $10 \%$ formalin for $8 \mathrm{~h}$ at room temperature and embedded in paraffin. Then the tissues were sectioned $(1.5 \mathrm{~mm}$ thick) and deparaffinized in xylene twice for 5 min each; hydrated with $100 \%$ ethanol twice for 3 min each; hydrated with $95 \%$ ethanol for $1 \mathrm{~min}$; and Rinsed in distilled water. The experimental method was as described previously (11) and sections $\left(1.5 \mathrm{~mm}\right.$ thick) were incubated at $4^{\circ} \mathrm{C}$ overnight with the rabbit anti-human CLDN12 (cat. no. ab107061; Abcam, Cambridge, UK) and E-Cadherin (cat. no. 3195; Cell Signaling Technology, Inc., Danvers, MA, USA) at a dilution of 1:400. Sections were then incubated with serum (provided by the UltraSensitive $^{\mathrm{TM}}$ SP IHC kit; cat. no. KIT-9710; Fuzhou Maixin Biotech co., Ltd., Fuzhou, China) for $30 \mathrm{~min}$ at $20^{\circ} \mathrm{C}$ to block the non-specific binding of immunoglobulin. Subsequently, slides were incubated with the goat anti-rabbit amplification reagent (provided by the UltraSensitive ${ }^{\mathrm{TM}}$ SP IHC kit) for $30 \mathrm{~min}$ at room temperature. Samples were then incubated with DAB for $5 \mathrm{~min}$ at room temperature. For negative controls, tissue sections were incubated with rabbit polyclonal antibodies against claudin-2 (cat. no. ab53032; 1:400; Abcam) at $4^{\circ} \mathrm{C}$ overnight. All sections were scored by two pathologists using a light microscope (E100, Nikon Corporation, Tokyo, Japan; magnification, x400). The expression of CLDN12 and E-Cadherin that were localized at the cell membrane were considered positive. The staining and scoring of CLDN12 and E-Cadherin protein expression levels were classified semi-quantitatively based on the total combined scores of the percentage of positively stained tumor cells together with the staining intensity as previously described (12).

Follow-up. Patients were followed from the beginning of diagnosis up to 60 months to evaluate the extent of metastasis and to determine survival times. Survival time was calculated from the beginning of diagnosis to the time of death or loss to follow-up. By the end of May 2017, all patients had received follow-up either on an outpatient basis or by telephone interview. The living status of each patient was confirmed. Survival analysis was conducted through use of Kaplan-Meier curves with the Log-Rank test.

Cell culture. The human embryonic kidney cells 293T cells, the human bronchial epithelial cell line BEAS-2B and the SqCC cell lines H226 and SK-MES-1 were purchased from the Shanghai Cell Bank of the Chinese Academy of Sciences (Shanghai, China) and cultured in Dulbecco's modified Eagle's medium (DMEM) supplemented with $10 \%$ fetal bovine serum (FBS) at $37^{\circ} \mathrm{C}$ in a humidified incubator containing $5 \% \mathrm{CO}_{2}$.

Plasmid construction and transfection. A total of $3 \mathrm{mg}$ of eukaryon expression vector pNSE-IRES2-EGFP (+)-CLDN12 (GeneBank accession no. NM022890) was synthesized and amplified by Nanjing KeyGen Biotech Co. Ltd. (Nanjing, China). The final construct was confirmed by direct sequence analysis. A total of $5 \mu \mathrm{g}$ eukaryon expression vector pNSE-IRES2-EGFP (+)-CLDN12 (1000 ng/ $\mu$ l) was transfected into cells using the SuperFect Transfection Reagent (Takara Bio, Inc., Otsu, Japan). Clones resistant to G418 (Sigma-Aldrich; Merck KGaA, Darmstadt, Germany) were expanded in culture at $37^{\circ} \mathrm{C}$ in a humidified incubator containing $5 \% \mathrm{CO}_{2}$ as a monoclonal population for 3 weeks. A monoclonal strain of BEAS-2B transfected with the p- IRES2-EGFP (+)-CLDN12 plasmid and a monoclonal strain of BEAS-2B transfected with p-NSE-IRES2-EGFP plasmid (1000 ng/ $\mu \mathrm{l})$ were obtained, which were termed as BEAS-CLDN12 and BEAS-Vector. Cells transfected with the empty vector pNSE-IRES2-EGFP1-C1 (+) (Nanjing KeyGen Biotech Co. Ltd., Nanjing, China) were used as a vector control. Cells were collected and seeded for subsequent experimentation at $48 \mathrm{~h}$ post-transfection.

Western blotting. Rabbit anti-human E-Cadherin (cat. no. 3195), rabbit anti-human phospho(p)-signal transducer and activator of transcription 1 (Stat1; cat. no. 7649), rabbit anti-human Stat1 antibody (cat. no. 14994), rabbit anti-human 
tyrosine kinase 2 (Tyk2; cat. no. 13531) and rabbit anti-human Vimentin (cat. no. 5741) antibodies were purchased from Cell Signaling Technology, Inc. Rabbit polyclonal antibodies against CLDN12 (cat. no. ab107061) and mouse anti-human $\beta$-actin (cat. no. ab8227) were purchased from Abcam. Total protein was extracted from cells of the respective groups using a protein extraction reagent (Beijing Biolab Technology Co., Ltd., Beijing, China) following culture at $37^{\circ} \mathrm{C}$ in a humidified incubator containing $5 \% \mathrm{CO}_{2}$ for $48 \mathrm{~h}$. The protein concentration of cell lysates was determined using a BCA protein assay kit (Pierce; Thermo Fisher Scientific, Inc., Waltham, MA, USA). Following this, $30 \mu \mathrm{g}$ of total protein per lane was separated via 10\% SDS-PAGE and transferred onto nitrocellulose membranes (EMD Millipore, Billerica, MA, USA). Membranes were then blocked with $4 \%$ skimmed milk powder for $2 \mathrm{~h}$ at room temperature and incubated with the aforementioned primary antibodies (all 1:1,000) against E-Cadherin, p-Stat1, Stat1, Tyk2, Vimentin, CLDN12 and $\beta$-actin overnight at $4^{\circ} \mathrm{C}$. Samples were washed and incubated with horseradish peroxidase-conjugated goat anti-rabbit immunoglobulin G antibodies (1:1,000; ab6721, Abcam) for $2 \mathrm{~h}$ at room temperature. Immunoreactive bands were detected using enhanced chemiluminescent western blotting reagents (GE Healthcare, Chicago, IL, USA) and analyzed using Image Lab 6.0.1 software (Bio-Rad Laboratories, Inc., Hercules, CA, USA).

Immunofluorescence method. Cells were washed three times with PBS, fixed with $4 \%$ paraformaldehyde for $10 \mathrm{~min}$ at room temperature, permeabilized with $0.1 \%$ Triton $\mathrm{X}-100$ (cat. no. 9002-93-1; Sigma-Aldrich; Merck KGaA) and blocked with $2 \%$ bovine serum albumin (Changchun Bote Biological Technology Co., Ltd., Changchun, China) in PBS for $1 \mathrm{~h}$ at room temperature. Staining for immunofluorescence was performed with the following primary antibodies: Rabbit anti-human CLDN12, rabbit anti-human E-Cadherin and rabbit anti-human Vimentin, which were diluted in blocking solution $(1: 1,000)$ and incubated for $30 \mathrm{~min}$ at room temperature. Subsequently, cells were incubated with Alexa Fluor 647-conjugated anti-rabbit IgG antibodies (cat. no. ab150093; Santa Cruz Biotechnology, Inc.; 1:1,000). Images were captured using a fluorescence microscope (Nikon Eclipse TE2000-S, Nikon Corporation; magnification, x400).

Cell Counting kit-8 (CCK-8) assay. A cell proliferation curve was determined using a colorimetric water-soluble tetrazolium salt assay (CCK-8; Dojindo Molecular Technologies, Inc., Kumamoto, Japan) according to the manufacturer's protocol. Cells were seeded into 96 -well plates at a density of $2 \times 10^{5}$ cells/well in triplicate and cultured overnight at $37^{\circ} \mathrm{C}$. Cell proliferation was recorded every $12 \mathrm{~h}$ for 4 days. The absorption of cell solution was measured at a wavelength of $450 \mathrm{~nm}$.

Wound-healing assay. Cells were cultured as a monolayer to $70 \%$ confluence on gridded plastic dishes and wounded by scratching with a $100-\mu 1$ pipette tip. Following this, cells were washed three times with PBS. The wounds were photographed with a light microscope (E100; Nikon Corporation; magnification, x200) at the same field of view at 0,12 and $24 \mathrm{~h}$, respectively.
Transwell invasion assay. Cells were cultured in DMEM (Gibco; Thermo Fisher Scientific, Inc., Waltham, MA, USA) with $10 \%$ FBS to a $90 \%$ confluence, then cultured at in FBSfree medium at $37^{\circ} \mathrm{C}$ in a humidified incubator containing $5 \% \mathrm{CO}_{2}$ for $24 \mathrm{~h}$. The medium containing chemotactic factors from the cell culture was collected. For the cell invasion assay, $3 \times 10^{4}$ cells suspended in $100 \mu \mathrm{FBS}$-free medium were added into the upper chamber of transwell chambers (8-mm pore size; BD Biosciences, Franklin Lakes, NJ, USA) coated with Matrigel (cat. no. 356234; BD Biosciences) and incubated at $37^{\circ} \mathrm{C}$ for $30 \mathrm{~min}$. A total of $600 \mu \mathrm{l}$ the medium with chemotactic factors containing $10 \%$ FBS was added into the lower chambers. Cells were incubated at $37^{\circ} \mathrm{C}$ in a humidified $5 \% \mathrm{CO}_{2}$ atmosphere for $6 \mathrm{~h}$. Any cells that remained on the upper chamber were removed carefully with a cotton swab. Migrated or invaded cells that had traversed the membrane were fixed in $95 \%$ methanol for $10 \mathrm{~min}$ at room temperature and stained with $0.5 \%$ crystal violet for $10 \mathrm{~min}$ at room temperature. The cells were subsequently photographed and counted in five randomly selected visual fields under an inverted microscope (Olympus Corporation; magnification, $\mathrm{x} 200$ ).

RNA interference (RNAi). Frozen glycerol bacterial stocks containing pGCSIL-scramble and pGCSIL-Tyk2-RNAi were purchased from Nanjing KeyGen Biotech Co., Ltd. pGCSIL-scramble was used as the control insert sequence. Tyk2-RNAi (Target ID, 29473) was the targeting sequence. $293 \mathrm{~T}$ cells $\left(0.2 \times 10^{7}\right.$ per well $)$ were seeded and maintained for $24 \mathrm{~h}$ to achieve $70-80 \%$ confluence in 6-well dishes (Cor ning Costar Co., Cambridge, MA, USA). The plasmids, including pGCSIL-Tyk2-RNAi or pGCSIL-scramble, $5 \mu \mathrm{g}$ of the packaging vector pHelper 1.0 and $5 \mu \mathrm{g}$ of a vesicular stomatitis virus glycoprotein expression plasmid vector, were added to Opti-MEM (Corning Costar Co.), to a final volume of $1.0 \mathrm{ml}$. Following this, $50 \mu \mathrm{l}$ of Lipofectamine (Invitrogen; Thermo Fisher Scientific, Inc.) was added to $950 \mu$ FBS-free DMEM (Gibco; Thermo Fisher Scientific, Inc., Waltham, MA, USA). These two solutions were mixed and combined with the cells. Lentiviral particles were harvested $48 \mathrm{~h}$ following transfection, and the viral titer was determined using counting green fluorescent protein-expressing cells under a fluorescence microscope (Nikon Diaphot 300, Nikon Instruments, Inc., Tokyo, Japan) with filters at $96 \mathrm{~h}$ post-transfection. The BEAS-2B-CLDN12 cells which were transfected with the pGCSIL-scramble and pGCSIL-Tyk2-RNAi plasmids were termed as BEAS-Scramble or BEAS-siRNA groups.

Statistical analysis. All experiments were repeated three times and all data were presented as the mean \pm standard deviation of at least three experimental results. Comparisons between two groups were made using a Student's t-test. One-way analysis of variance followed by a Dunnett's multiple comparisons test were used for multiple group comparisons. Kaplan-Meier analysis was used for survival analysis in patients with SqCC. Additionally, the $\chi^{2}$ test was applied to assess the association of clinical case indicators. $\mathrm{P}<0.05$ was considered to indicate a statistically significant difference. 
Table I. Expression of CLDN12 and clinicopathological characteristics of the patients with lung squamous cell carcinoma.

\begin{tabular}{|c|c|c|c|c|}
\hline Item & $\mathrm{n}$ & CLDN12 (+) & CLDN12 (-) & P-value \\
\hline Tumor tissue & 50 & 32 & 18 & $<0.001$ \\
\hline Normal mucosa & 20 & 5 & 15 & \\
\hline Age (years) & & & & 0.794 \\
\hline$\leq 60$ & 22 & 14 & 8 & \\
\hline$>60$ & 28 & 18 & 10 & \\
\hline Smoker & & & & 0.372 \\
\hline Yes & 30 & 17 & 13 & \\
\hline No & 20 & 15 & 5 & \\
\hline E-Cadherin & & & & 0.001 \\
\hline+ & 29 & 13 & 16 & \\
\hline- & 21 & 19 & 2 & \\
\hline Histological grade & & & & 0.812 \\
\hline Well and moderately differentiated & 24 & 15 & 9 & \\
\hline Poorly differentiated & 26 & 17 & 9 & \\
\hline Lymph node metastasis & & & & 0.001 \\
\hline+ & 27 & 21 & 6 & \\
\hline- & 23 & 11 & 12 & \\
\hline Grade & & & & 0.736 \\
\hline I-II & 35 & 22 & 13 & \\
\hline III-IV & 15 & 10 & 5 & \\
\hline
\end{tabular}

P-values were determined with the $\chi^{2}$ test. CLDN12, claudin-12.

\section{Results}

Expression of CLDN12 in human SqCC tissues is upregulated and negatively associated with E-Cadherin. CLDN12 expression was assessed in the 50 specimens of SqCC tissue, 10 specimens of lung adenocarcinoma tissue and 20 specimens of non-neoplastic mucosal tissue (Fig. 1). As indicated in Table I, high-level expression of CLDN12 was detected in $64.0 \%$ $(32 / 50)$ of the SqCC tissues and in $25.0 \%(5 / 20)$ of the normal mucosal tissues $(\mathrm{P}=0.0002)$. The associations of CLDN12 with clinical pathological indicators were analyzed, and it was revealed that the expression of CLDN12 was not associated with age $(\mathrm{P}=0.794)$, smoking status $(\mathrm{P}=0.372)$, degree of differentiation $(\mathrm{P}=0.812)$ or clinical staging $(\mathrm{P}=0.736)$, but was associated with lymph node metastasis $(\mathrm{P}=0.0001)$ and the expression of E-Cadherin $(\mathrm{P}=0.0001$; Table I). These data revealed that the expression of CLDN12 was increased in SqCC tissues and was associated with lymph node metastasis and E-Cadherin expression.

Association with survival and clinical outcomes. The length of follow-up ranged from 8 to 60 months. Kaplan-Meier survival curves and the Log-Rank test were used to determine whether there was an association between CLDN12 and survival time. As indicated in Fig. 2, patients with tumors that were positive for CLDN12 protein (median survival, 44.85 months) had a significantly shorter survival time than those whose tumors were negative for CLDN12 protein (median survival, 55.23 months; $\left.\chi^{2}=7.176, \mathrm{P}=0.0074\right)$.
Protein expression of CLDN12 in SqCC cells and tissues. Analysis of the semiquantitative immunoblotting results was performed to estimate and compare the statistical differences in the expression of CLDN12 among the human bronchial epithelial cell line BEAS-2B and SqCC cell lines H226 and SK-MES-1. As depicted in Fig. 3, the protein expression levels of CLDN12 were significantly increased in the SqCC cell lines H226 and SK-MES-1 compared with the human bronchial epithelial cell line BEAS-2B $(\mathrm{P}<0.01)$.

Semiquantitative immunoblotting analysis was also performed to determine the statistical differences in CLDN12 expression among 3 cases of SqCC tissues and 3 cases of non-neoplastic mucosal tissues, which were drawn from the 50 cases of SqCC tissues and 20 cases of non-neoplastic mucosal tissues at random. As indicated in Fig. 3, the protein expression levels of CLDN12 were significantly upregulated in the SqCC tissues compared with the non-neoplastic mucosal tissues $(\mathrm{P}<0.01)$.

The impact of CLDN12 on human bronchial epithelial cell EMT.Previous findings revealed that the expression of CLDN12 was low or absent in the human bronchial epithelial cell line BEAS-2B. To detect the impact of CLDN12 on the metastasis of BEAS-2B cells, a p-EGFP-C1/CLDN12 plasmid was transfected into the cells. Following G418 screening, a monoclonal strain of BEAS-2B transfected with p-EGFP-C1/CLDN12 plasmid and a monoclonal strain of BEAS-2B transfected with p-EGFP-C1/vector plasmid was obtained, which were termed as BEAS-CLDN12 and BEAS-vector. 
A

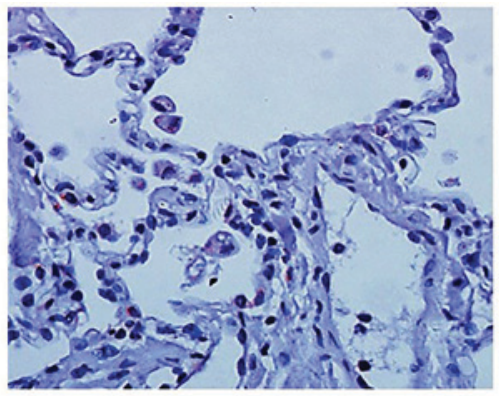

C

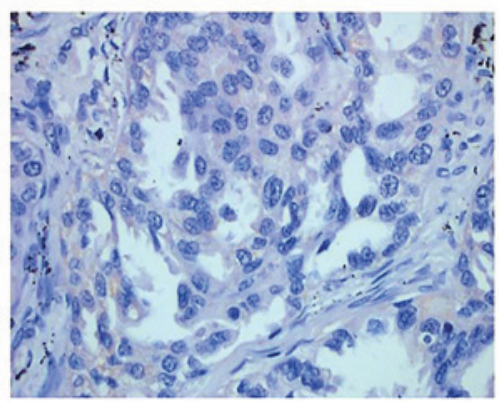

E

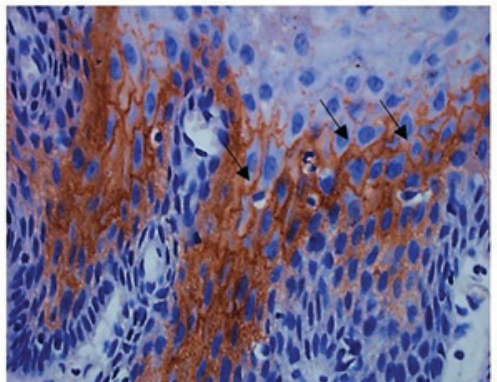

B

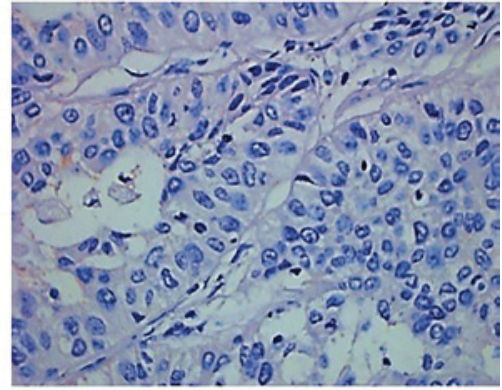

D

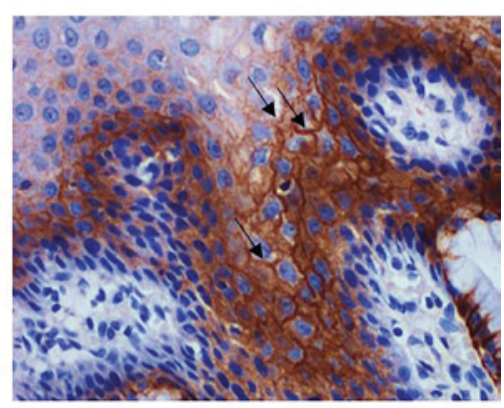

F

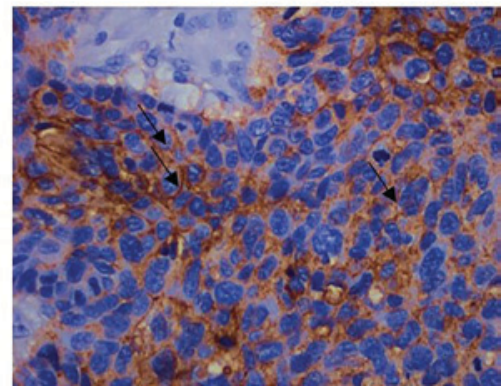

Figure 1. Expression of CLDN12 in human lung carcinoma and non-neoplastic mucosal tissues. (A) Low-level expression of CLDN12 in the non-neoplastic mucosal tissues. (B) Low-level expression of CLDN12 in well-differentiated lung adenocarcinoma tissues. (C) Low-level expression of CLDN12 in poorly differentiated lung adenocarcinoma tissues. (D) High-level expression of CLDN12 in well differentiated SqCC tissues. (E) High-level expression of CLDN12 in moderately differentiated SqCC tissues. (F) High-level expression of CLDN12 in poorly differentiated SqCC tissues. SqCC, squamous cell carcinoma; CLDN12, claudin-12. Magnification, x400..

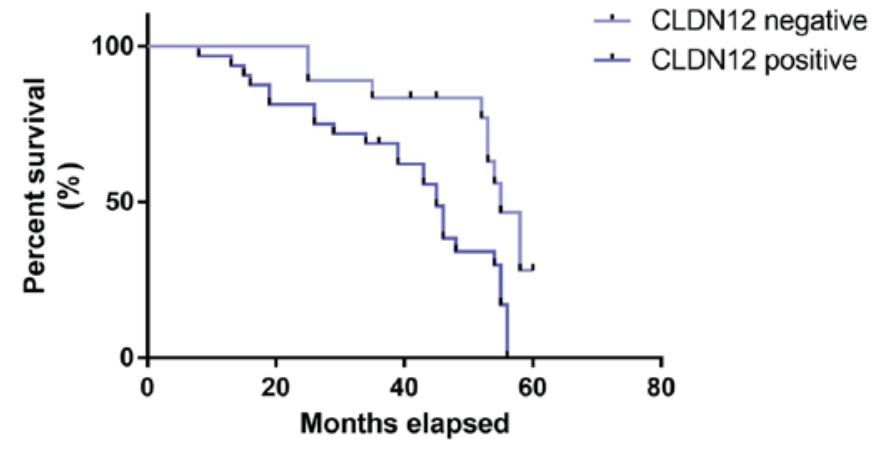

Figure 2. Association between the expression of CLDN12 and survival time of patients with squamous cell carcinoma. Kaplan-Meier survival curves and the Log-Rank test were used in analysis of association between CLDN12 and survival time $\left(\chi^{2}=7.176, P=0.0074\right)$. CLDN12, claudin-12.

Western blotting was used to detect the expression of CLDN12,E-Cadherin and Vimentin in the BEAS-2B cells. The results revealed that CLDN12 and Vimentin protein expression levels in the BEAS-CLDN12 cells were significantly increased compared with those in the empty vector group, whereas the protein expression levels of E-Cadherin in the clonal BEAS-2B cells was significantly decreased compared with the empty vector group ( $\mathrm{P}<0.01$; Fig. $4 \mathrm{~A}$ and $\mathrm{B})$.

Immunofluorescence was used to detect the expression and localization of CLDN12, E-Cadherin and Vimentin in BEAS-2B cells. The results suggested that CLDN12 expression levels in the CLDN12 overexpressed group were markedly increased compared with the empty vector group, and that CLDN12 was primarily localized on cell membranes. Conversely, the expression levels of E-Cadherin on the membranes of the CLDN12 overexpressed group were decreased compared with the empty vector group. Furthermore, the expression levels of Vimentin in the cytoplasm of the CLDN12 overexpressed group were increased compared with the empty vector group (Fig. 4C).

These results demonstrated that BEAS-2B clones stably expressing CLDN12 were successfully established, and that cells transitioned from an epithelial phenotype to interstitial phenotype following the overexpression of CLDN12; i.e., the EMT process was promoted in these BEAS-2B cells.

Impact of CLDN12 on the proliferation rate and metastasis ability of human bronchial epithelial cells. Growth curves of BEAS-2B cells were determined using the CCK-8 method. As 
A
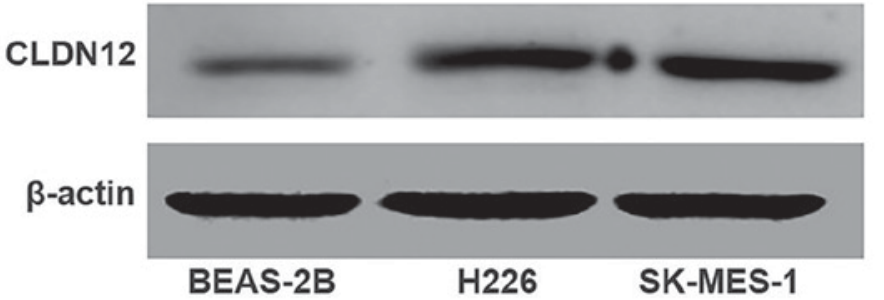

C

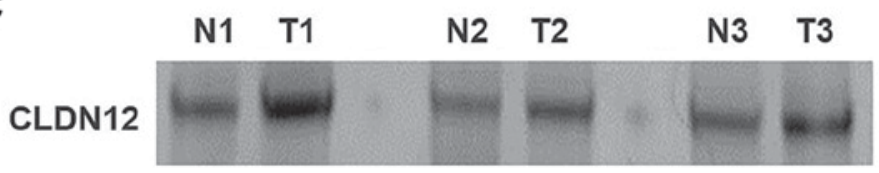

$\beta$-actin

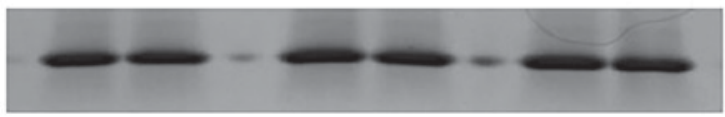

B
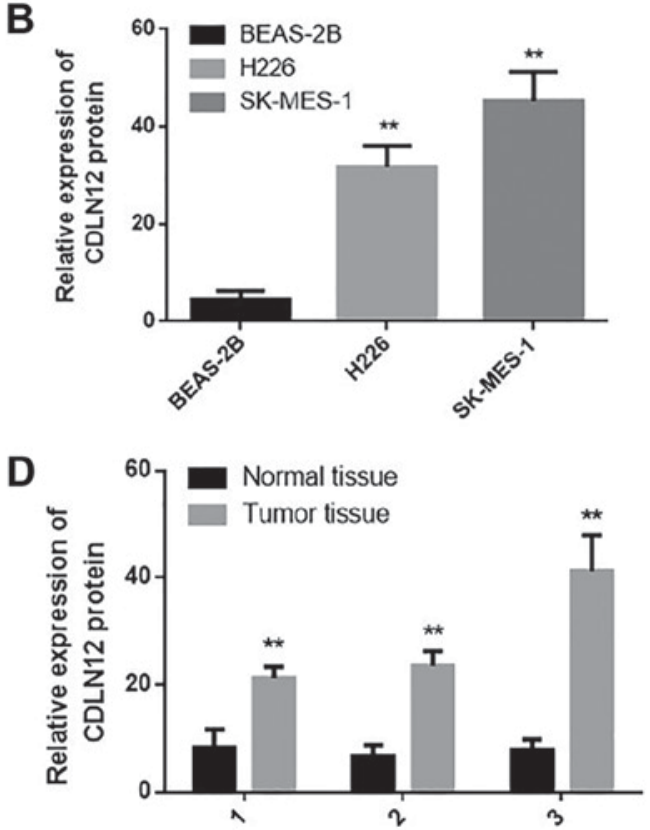

Figure 3. Protein expression of CLDN12 in human SqCC cells and tissues. (A) Protein expression of CLDN12 in SqCC cell lines and the human bronchial epithelial cell line BEAS-2B. (B) Statistical differences in the protein expression levels of CLDN12 between SqCC and bronchial epithelial cells. One-way analysis of variance and Dunnett's multiple comparisons test were performed. ${ }^{* *} \mathrm{P}<0.01$ vs. BEAS-2B cells. (C) Protein expression of CLDN12 in SqCC tissues and non-neoplastic mucosae. (D) Statistical differences in the protein expression level of CLDN12 between SqCC tissues and non-neoplastic mucosae. Student's t-test was performed. ${ }^{* *} \mathrm{P}<0.01$ vs. non-neoplastic normal lung tissues. CLDN12, claudin-12; SqCC, squamous cell carcinoma.

revealed in Fig. 5A, the proliferation rate of BEAS-CLDN12 cells was significantly higher than that of the empty vector group at 36,48 and $72 \mathrm{~h}(\mathrm{P}<0.01)$.

A wound-healing experiment was used to detect the impact of CLDN12 on the migratory ability of human bronchial epithelial cells. The results indicated that at 12 and $24 \mathrm{~h}$, the migration distances of BEAS-CLDN12 cells were significantly greater compared with those of the empty vector group ( $\mathrm{P}<0.01$; Fig. 5B). Additionally, the Transwell invasion assay was used to assess invasive ability in the human bronchial epithelial cells. At $6 \mathrm{~h}$ after the cells were seeded, those cells that invaded under the membrane of the chamber were observed. The results demonstrated that the number of invasive BEAS-CLDN12 cells was increased compared with the empty vector group (Fig. 5C). Statistical analysis revealed the difference was significant $(\mathrm{P}<0.01$; Fig. 5D). These results suggested that CLDN12 significantly promoted the proliferation and metastasis of BEAS-2B cells in vitro.

Impact of Tyk2/Stat1 signaling pathway activation on the invasion and migration abilities of BEAS-2B cells. Western blotting was utilized to investigate the activation state of the Tyk2/Stat1 signaling pathway. Following the overexpression of CLDN12, the phosphorylation levels of Stat1 were significantly increased in BEAS-2B cells compared with the control $(\mathrm{P}<0.01$; Fig. 6A and B). To determine the impact of the Tyk2/Stat1 signaling pathway on the invasion and migration abilities of BEAS-2B cells, pGCSIL-scramble and pGCSIL-Tyk2-RNAi plasmids were transfected into BEAS-2B-CLDN12 cells. Following the knockdown of Tyk2, the phosphorylation levels of Stat 1 were significantly downregulated in the BEAS-2B cell line overexpressing CLDN12 ( $\mathrm{P}<0.01$; Fig. 6A and B). Furthermore, the expression levels of E-Cadherin in
Tyk2-RNAi cells were increased and the expression of levels of Vimentin in Tyk2-RNAi cells were significantly decreased $(\mathrm{P}<0.01$; Fig. 6A and $\mathrm{B})$.

A Transwell chamber assay and wound-healing assay were also used to analyze the effect of Tyk 2 on the invasive and migratory abilities of the examined cells. The results demonstrated that the number of invasive BEAS-2B cells in the CLDN12 overexpressed group was significantly decreased following Tyk2 silencing $(\mathrm{P}<0.01$; Fig. 6C and D). The migration distances of the Tyk2-RNAi cells were significantly decreased compared with those of the scramble group at 12 and $24 \mathrm{~h}(\mathrm{P}<0.01$; Fig. 6E).

\section{Discussion}

Certain reports have indicated CLDN to function as a tumor suppressor gene, where loss of CLDN contributed to enhanced tumorigenic properties of tumor cells $(13,14)$. In one study, expression of CLDN1 was identified to be reduced in stage II and III rectal cancer and was established as a factor that significantly correlated with recurrence and poor prognosis (15). Furthermore, the expression of CLDN6 has been revealed to be downregulated in cervical carcinoma tissues, whereas the gain of CLDN6 expression suppressed cell proliferation and the colony formation capacity of cervical carcinoma cells in vitro, and tumor growth in vivo (12). However, in contrast to these results, increasing evidence suggests that CLDNs may serve as pro-oncogenes in various types of human cancer. For instance, it was highlighted that CLDN1 had a key role in inflammation-induced growth and progression of colorectal carcinoma (16). Furthermore, Philip et al (17) reported that CLDN7 expression in colorectal cancer contributed to cell motility and invasion. Therefore, specific CLDNs may have differential impacts on the biological 
A

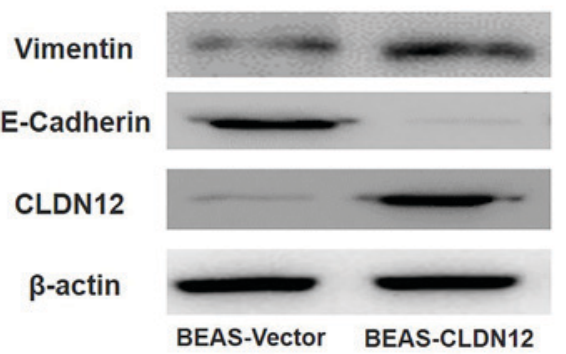

B

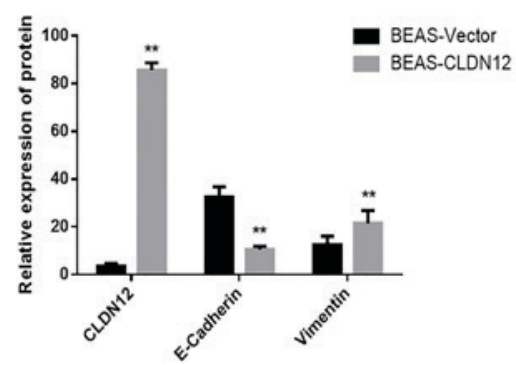

C
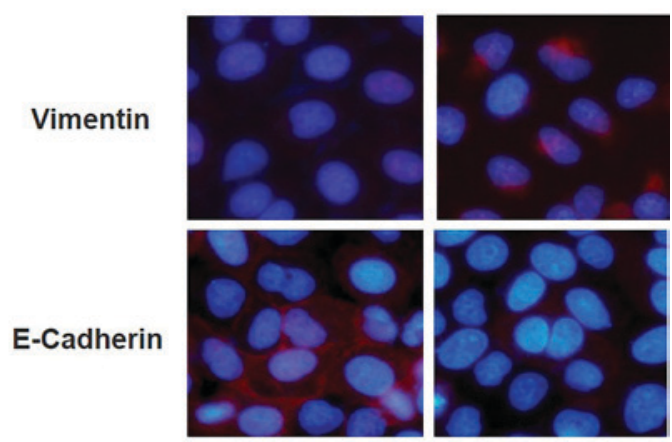

CLDN12
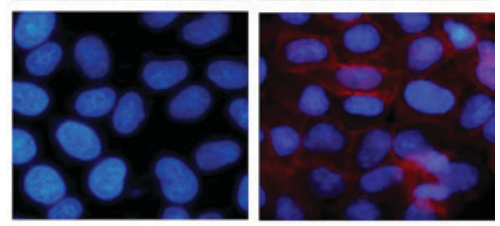

BEAS-Vector

Figure 4. Impact of CLDN12 on the epithelial-mesenchymal transition of human bronchial epithelial cells. (A) Expression of CLDN12, E-Cadherin and Vimentin in BEAS-2B cells; (B) corresponding statistical analysis of protein expression levels. (C) Localizations of CLDN12, E-Cadherin and Vimentin in BEAS-2B cells (magnification, $\mathrm{x} 400$ ). Student's t-test was performed. ${ }^{* *} \mathrm{P}<0.01$ vs. empty vector group. CLDN12, claudin-12; SqCC, squamous cell carcinoma; E-Cadherin, epithelial-Cadherin.

A

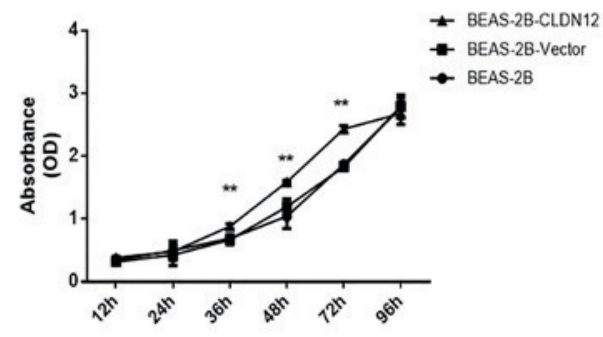

B

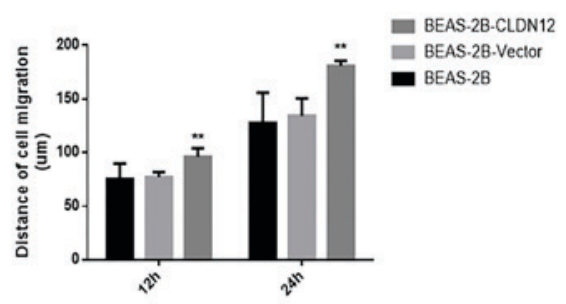

C

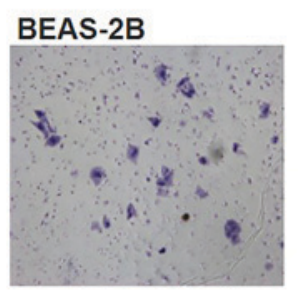

BEAS-2B-Vector

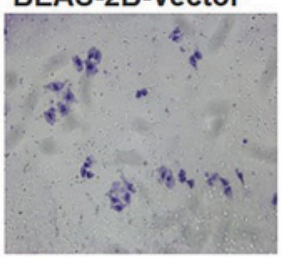

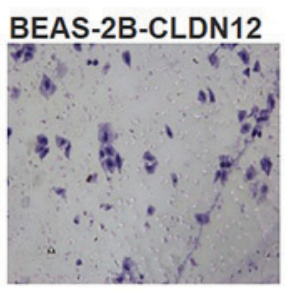

D

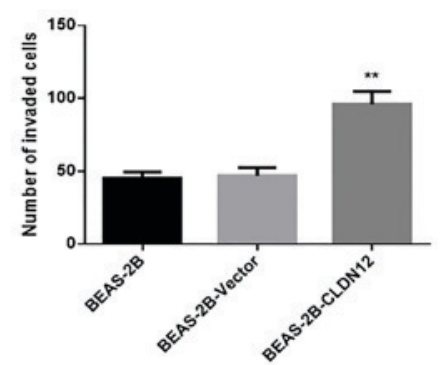

Figure 5. Impact of CLDN12 on the proliferation and metastasis abilities of cells in vitro. (A) Growth curve of BEAS-2B cells determined by the Cell Counting kit-8 method. (B) Migration ability of the BEAS-2B cell line in vitro determined by wound healing assays. (C) Invasive ability of the BEAS-2B cell line in vitro determined by the Transwell chamber method (magnification, x200); (D) corresponding statistical analysis of invaded cell numbers. Analysis of variance and Dunnett's multiple comparisons test was performed. ${ }^{* *} \mathrm{P}<0.01$ vs. empty vector group. CLDN12, claudin- 12 .

behavior of a given tumor (18-20). One potential reason for the discrepancy in results may be that the function of CLDNs is specific and relies on different interacting molecules in various cells $(21,22)$.

Recently, a number of studies have focused on the role of CLDNs in the tumorigenesis of human lung carcinoma. For instance, the expression of CLDN1 was identified as a positive prognostic factor in cases of SqCC (23). Notably, CLDN2 has also been indicated to be overexpressed in human lung adenocarcinoma tissues and a novel target in lung adenocarcinoma (24). Additionally, CLDN3 was reported to inhibit the metastatic phenotype of SqCC via suppression of the Wnt/ $\beta$-catenin signaling pathway (25). Other studies have revealed that downregulation of CLDN7 has been reported to 
A
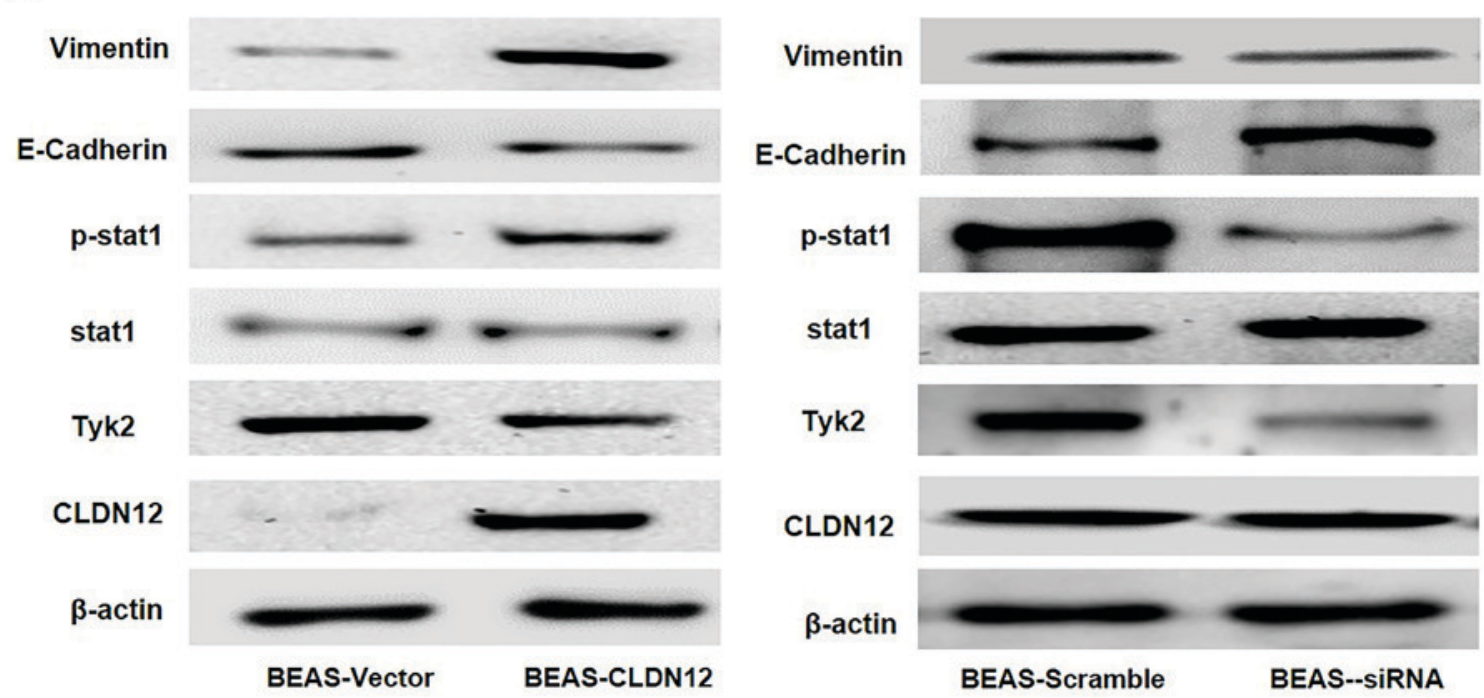

B
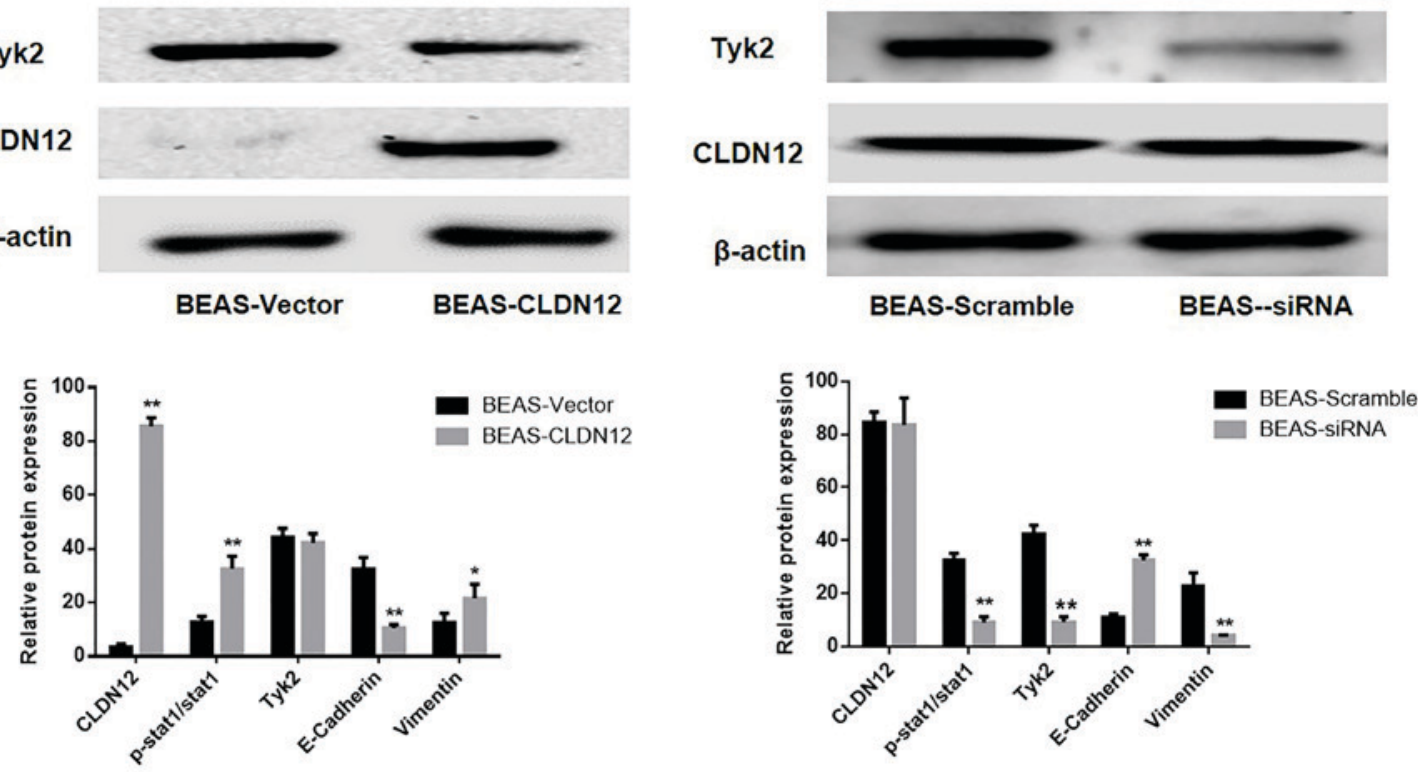

C
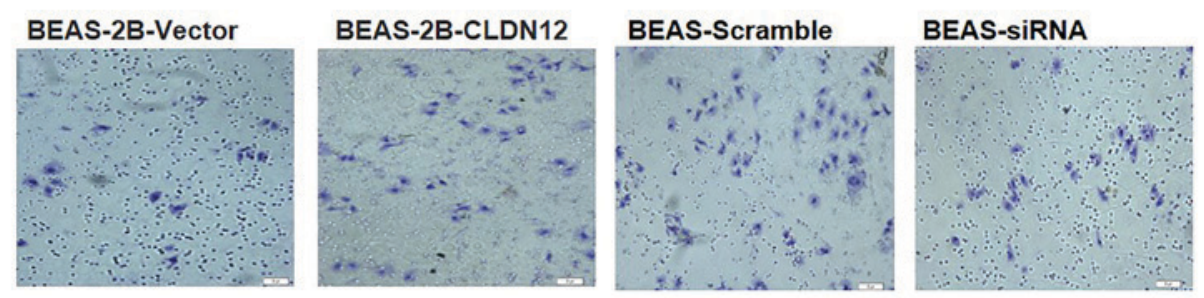

E
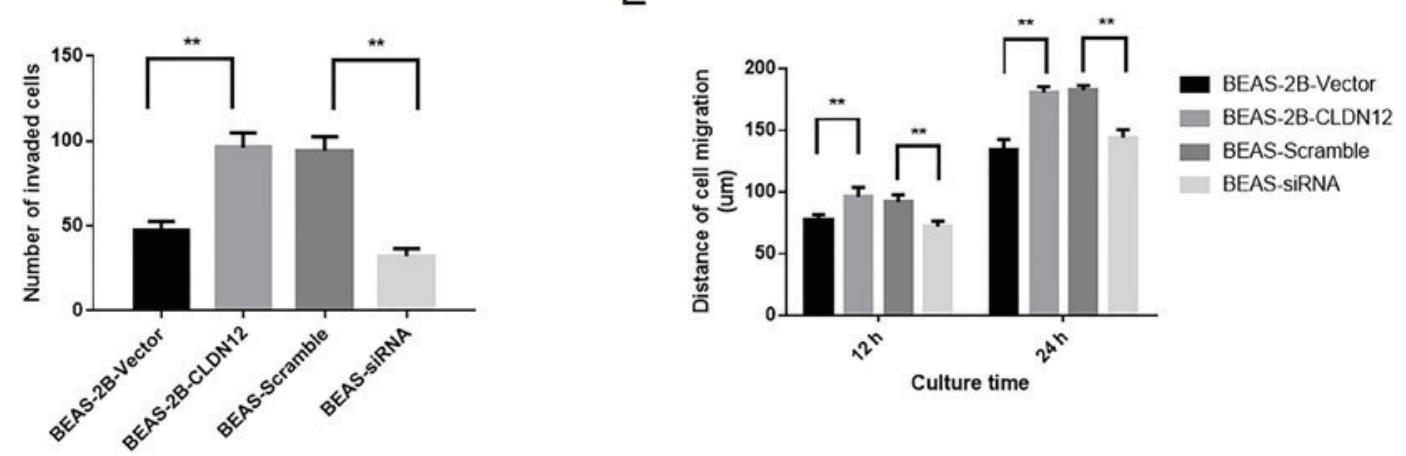

Figure 6. Impact of Tyk2/Stat1 signaling pathway activation on the epithelial-mesenchymal transition process of BEAS-2B cells. RNA interference was used to silence Tyk2 expression in CLDN12-expressing cells. (A) Western blot analysis was used to examine the effects of silencing Tyk2, the activation of the Stat1 signaling pathway, E-Cadherin expression and Vimentin expression in the BEAS-2B cell line. (B) Corresponding statistical analysis of the protein expression levels. (C) The Transwell chamber method was used to detect the impact of Tyk2 silencing on the invasive ability of cells in vitro (magnification, x200). (D) Corresponding statistical analysis of invasive cells. (E) The wound-healing assay was used to detect the migration ability of the BEAS-2B cell line in vitro. Analysis of variance and Dunnett's multiple comparisons test was performed. " $\mathrm{P}<0.05$ and ${ }^{* *} \mathrm{P}<0.01$ vs. BEAS-2B CLDN12 group or as indicated. Tyk2, tyrosine kinase 2; Stat1, signal transducer and activator of transcription 1; E-Cadherin, epithelial-Cadherin.

promote the survival capacity of lung cancer cells under the hypoxic conditions of the tumor microenvironment $(26,27)$.
CLDN12 is among the 27 members of the CLDN protein family, and current understanding of the biological function 
of CLDN12 is primarily limited to its role in epithelial and epidermal permeability, barrier protection and cell connections, with limited reports on the association between CLDN12 and tumors (28). The present data suggested that CLDN12 expression was upregulated in SqCC, not in lung adenocarcinoma, and was involved with the lymph node metastasis of SqCC. Additionally, the association between CLDN12 and the expression level of E-Cadherin in SqCC was investigated. The results indicated that the expression of E-Cadherin was inversely associated with that of CLDN12. These data suggested that CLDN12 may be negatively associated with the expression of E-Cadherin during the tumorigenesis and progression of $\mathrm{SqCC}$, and therefore, that the combination of CLDN12 and E-Cadherin expression may be useful as an independent predictor for the diagnosis of SqCC as well as for the determination of distant metastasis and prognosis. To verify this hypothesis, a human bronchial epithelial cell line, BEAS-2B, that stably expressed CLDN12 was established. It was indicated that overexpression of CLDN12 significantly enhanced the metastasis and migratory abilities of this human bronchial epithelial cell line.

To date, certain studies have demonstrated that CLDN proteins can bind with various proteins associated with cellular signal transduction, and thereby regulate a series of cell behaviors, including the EMT process (29). For instance, Philip et al (17) reported that CLDN7 expression in colorectal cancer contributed to motility and invasion by promoting a shift towards EMT through recruiting epithelial cell adhesion molecule towards TACE/presenilin2. However, to date, there have been few reports on the roles of CLDN12 in human tumors, and the specific molecular mechanisms involved remain to be clarified. In the present study, a preliminary investigation of the molecular mechanism associated with the effect of CLDN12 on the metastasis ability of bronchial epithelial cells was performed. The present study identified that CLDN12 upregulation affected the Stat1 signaling pathway via Tyk2, and ultimately enhanced the EMT of the human bronchial epithelial cell line BEAS-2B in vitro. To date, few studies have indicated that Tyk2/Stat1 signaling impacts the role of various CLDNs in human tumorigenesis. One of the most interesting findings in the present research is that upregulated CLDN12 contributed to an enhancement of cell invasion and migration through the Tyk2/Stat1 signaling pathway in SqCC. Considering the limited therapeutic options for patients with SqCC, the role of CLDN12 as a therapeutic target merits further investigation.

In the present study, it was suggested that CLDN12 may be a protooncogene in $\mathrm{SqCC}$, as the overexpression of CLDN12 significantly enhanced the metastasis ability of the human bronchial epithelial cell line BEAS-2B. Furthermore, the induction of Tyk2/Stat1 signaling appeared an important mechanism by which CLDN12 promoted the metastatic phenotype of SqCC cells.

\section{Acknowledgements}

Not applicable.

\section{Funding}

No funding was received.

\section{Availability of data and materials}

The datasets used and/or analyzed during the present study are available from the corresponding author on reasonable request.

\section{Authors' contributions}

LS and LF performed the experiments and analyzed the data. JC contributed to the conception and design of the study. LF also revised the manuscript critically for important intellectual content. All authors read and approved the final manuscript.

\section{Ethics approval and consent to participate}

All procedures performed involving human participants were in accordance with the ethical standards of the institutional and/or national research committee and with the 1964 Helsinki declaration and its later amendments or comparable ethical standards. This present study is retrospective and patient consent was obtained at the time of data collection. Ethics approval (approval no. JLU00896 and JLU01125) was granted by the Ethics Committee of Jilin University.

\section{Patient consent for publication}

Not applicable.

\section{Competing interests}

The authors declare that they have no competing interests.

\section{References}

1. Tabaries $\mathrm{S}$ and Siegel PM: The role of claudins in cancer metastasis. Oncogene 36: 1176-1190, 2017.

2. Matsuda A and Katanoda K: Five-year relative survival rate of lung cancer in the USA, Europe and Japan. Jpn J Clin Oncol 43: 1287-1288, 2013.

3. Osanai M, Takasawa A, Murata M and Sawada N: Claudins in cancer: Bench to bedside. Pflugers Arch 469: 55-67, 2017.

4. Kwon MJ: Emerging roles of claudins in human cancer. Int J Mol Sci 14: 18148-18180, 2013.

5. Balda MS and Matter K: Tight junctions at a glance. J Cell Sci 121: 3677-3682, 2008

6. Escudero-Esparza A, Jiang WG and Martin TA: The Claudin family and its role in cancer and metastasis. Front Biosci (Landmark Ed) 16: 1069-1083, 2011.

7. Ouban A: Claudin-1 role in colon cancer: An update and a review. Histol Histopathol 33: 1013-1019, 2018.

8. Szasz MA: Claudins as prognostic factors of breast cancer. Magy Onkol 56: 209-212, 2012 (In Hungarian).

9. Martin de la Fuente L, Malander S, Hartman L, Jönsson JM, Ebbesson A, Nilbert M, Måsbäck A and Hedenfalk I: Claudin-4 expression is associated with survival in ovarian cancer but not with chemotherapy response. Int J Gynecol Pathol 37: 101-109, 2018.

10. Ahmad R, Kumar B, Chen Z, Chen X, Müller D, Lele SM, Washington MK, Batra SK, Dhawan P and Singh AB: Loss of claudin-3 expression induces IL6/gp130/Stat3 signaling to promote colon cancer malignancy by hyperactivating Wnt/ $\beta$-catenin signaling. Oncogene 36: 6592-6604, 2017.

11. Zhang X, Ruan Y, Li Y, Lin D, Liu Z and Quan C: Expression of apoptosis signal-regulating kinase 1 is associated with tight junction protein claudin-6 in cervical carcinoma. Int J Clin Exp Pathol 8: 5535-5541, 2015.

12. Zhang X, Ruan Y, Li Y, Lin D and Quan C: Tight junction protein claudin- 6 inhibits growth and induces the apoptosis of cervical carcinoma cells in vitro and in vivo. Med Oncol 32: 148, 2015. 
13. Ouban A and Ahmed A: Claudins in human cancer, A review. Histol Histopathol 25: 83-90, 2010.

14. Zavala-Zendejas VE, Torres-Martinez AC, Salas-Morales B, Fortoul TI, Montano LF and Rendon-Huerta EP: Claudin-6, 7, or 9 overexpression in the human gastric adenocarcinoma cell line AGS increases its invasiveness, migration, and proliferation rate. Cancer Invest 29: 1-11, 2011.

15. Yoshida T, Kinugasa T, Akagi Y, Kawahara A, Romeo K, Shiratsuchi I, Ryu Y, Gotanda Y and Shirouzu K: Decreased expression of claudin-1 in rectal cancer: A factor for recurrence and poor prognosis. Anticancer Res 31: 2517-2525, 2011.

16. Cherradi S, Ayrolles-Torro A, Vezzo-Vie N, Gueguinou N, Denis V,Combes E, BoissièreF, Busson M, Canterel-Thouennon L, Mollevi C, et al: Antibody targeting of claudin-1 as a potential colorectal cancer therapy. J Exp Clin Cancer Res 36: 89, 2017.

17. Philip R, Heiler S, Mu W, Buchler MW, Zoller M and Thuma F: Claudin-7 promotes the epithelial-mesenchymal transition in human colorectal cancer. Oncotarget 6: 2046-2063,2015.

18. D'Souza T, Agarwal R and Morin PJ: Phosphorylation of claudin-3 at threonine 192 by cAMP-dependent protein kinase regulates tight junction barrier function in ovarian cancer cells. J Biol Chem 280: 26233-26240, 2005.

19. D'Souza T, Indig FE and Morin PJ: Phosphorylation of claudin-4 by PKCepsilon regulates tight junction barrier function in ovarian cancer cells. Exp Cell Res 313: 3364-3375, 2007.

20. Li X, Li Y, Qiu H and Wang Y: Downregulation of claudin-7 potentiates cellular proliferation and invasion in endometrial cancer. Oncol Lett 6: 101-105, 2013.

21. Lu Z: Functions of claudin-7 in human lung cancer (unpublished $\mathrm{PhD}$ thesis). 2012.

22. Micke P, Mattsson JS, Edlund K, Lohr M, Jirström K, Berglund A, Botling J, Rahnenfuehrer J, Marincevic M, Pontén F, et al: Aberrantly activated claudin 6 and 18.2 as potential therapy targets in non-small-cell lung cancer. Int J Cancer 135: 2206-2214, 2014
23. Moldvay J, Fabian K, Jackel M, Németh Z, Bogos K, Furák J, Tiszlavicz L, Fillinger J, Döme B and Schaff Z: Claudin-1 protein expression is a good prognostic factor in non-small cell lung cancer, but only in squamous cell carcinoma cases. Pathol Oncol Res 23: 151-156, 2017.

24. Du W, Xu X, Niu Q, Zhang X, Wei Y, Wang Z, Zhang W, Yan J, $\mathrm{Ru}$ Y, Fu Z, et al: Spi-B-mediated silencing of claudin-2 promotes early dissemination of lung cancer cells from primary tumors. Cancer Res 77: 4809-4822, 2017.

25. Che J, Yue D, Zhang B, Zhang H, Huo Y, Gao L, Zhen H, Yang Y and Cao B: Claudin-3 inhibits lung squamous cell carcinoma cell epithelial-mesenchymal transition and invasion via suppression of the Wnt/ $\beta$-catenin signaling pathway. Int J Med Sci 15: 339-351, 2018.

26. Lu Z, Liu Y, Xu J, Yin H, Yuan H, Gu J, Chen YH, Shi L, Chen D and Xie B: Immunohistochemical quantification of expression of a tight junction protein, claudin-7, in human lung cancer samples using digital image analysis method. Comput Methods Programs Biomed 155: 179-187, 2018

27. Lu Z, Kim DH, Fan J, Lu Q, Verbanac K, Ding L, Renegar R and Chen YH: A non-tight junction function of claudin-7-Interaction with integrin signaling in suppressing lung cancer cell proliferation and detachment. Mol Cancer 14: 120, 2015.

28. Koval M: Claudin heterogeneity and control of lung tight junctions. Annu Rev Physiol 75: 551-567, 2013.

29. Kominsky SL: Claudins: Emerging targets for cancer therapy. Expert Rev Mol Med 8: 1-11, 2006. 